\title{
Modelling of the Usefulness of Carbon Nanotubes as Antiviral Compounds for Treating Alzheimer Disease
}

\author{
Hakim Al Garalleh \\ Department of Mathematical Science, College of Engineering, University of Business and Technology, Jeddah, Saudi Arabia \\ Email: h.algaralleh@ubt.edu.sa
}

How to cite this paper: Al Garalleh, $\mathrm{H}$. (2018) Modelling of the Usefulness of Carbon Nanotubes as Antiviral Compounds for Treating Alzheimer Disease. Advances in Alzheimer's Disease, 7, 79-92. https://doi.org/10.4236/aad.2018.73006

Received: June 27, 2018

Accepted: September 3, 2018

Published: September 6, 2018

Copyright $\odot 2018$ by author and Scientific Research Publishing Inc. This work is licensed under the Creative Commons Attribution International License (CC BY 4.0).

http://creativecommons.org/licenses/by/4.0/

\section{(c) (i) Open Access}

\begin{abstract}
The new generations of nano-devices successfully apply with great promise as drug carriers in the treatment of different diseases. The proposed model aims to determine the pharmacological targets and evaluate the bio-safety of usefulness of carbon nanotube conjugated with two different antiviral compounds, Acetylcholine and Ravastigmine, for treating Alzheimer disease. We also obtain the medicinal model mathematically to evaluate the interaction energy arising from encapsulation of each antiviral compound inside the single-walled carbon nanotube. Acetylcholine is modelled as two-connected spheres, while Ravastigmine has two possible structures which are an ellipsoid and cylinder, all interacting with the interior wall of single-walled carbon nanotubes with variant radii $r_{c}$. Our calculations show that the single-walled carbon nanotube of radius $r_{c}$ greater than $3.391 \AA$ that will accept both drugs which are quite closer to the recent findings.
\end{abstract}

\section{Keywords}

Carbon Nanotubes (CNTs), Alzheimer Disease (AD), Acetylcholine (ACh)

(Aricept), Ravastigmine (RAV) (Exelon), Mitochondria (MTCDH),

Lysosomes, Van Der Waals Interaction and Lennard-Jones Potential

\section{Introduction}

Research in nanobiotechnology has rapidly increased since the development of molecular dynamic simulations (MDSs) in 1980, X-ray crystallography and scanning tunneling microscope in 1982. Nanobiotechnology is an interdisciplinary area combining the molecular biological approach with the micronanotechnology. This combination aims to design and develop new devices by ma- 
nipulating at the nanoscale their size, shape, distinct properties. It also presents revolutionary opportunities by implying the creation of nano-materials, against the infection, cancer cells and cardiac disorder, designed to interact with targeted sites in the body at sub-cellular scales with a high degree of certainty. This has led the scientific researchers to find the lightest, strongest and most conductive carbon nano-materials that capable of transporting different biomolecules through their surfaces. Carbon nanodevices are a family of very small tubes which are wholly composed of carbon atoms, having diameter measured by nanometre level (one-billionth scale) which is about to ten-thousand times smaller than the human hair, such as peptides, fullerenes, nano-rods, nano-buds, graphenes and cylindrical carbon nanotubes. Carbon nanotubes (CNTs) are selective nanoparticle because of their huge potential, low solubility and toxicity, outstanding properties, maximum loading capability and extraordinary thermal conductivity [1] [2]. They are classified into two main sub-groups; multi-walled and single-walled CNTs (MWCNTS and SWCNTs). These nanotubes can be widely functionalized with proteins, bio-active peptides and drugs, and used to deliver their loads to the targeted cells, such as for treating the infected sites, inhibiting the growth of pathogens or attacking the cancer cells [3] [4].

CNTs have attracted a lot of interesting researches since their discovery [5] [6]. Due to their unusual properties that can be practically modified with different bio-molecules through several techniques [4] [7]. There have been several studies addressed the ability of CNTs to conjugate with a wide variety of drugs for treatment purposes. CNTs have been explored for disease therapy applications, especially for cancer treatment [8]. In addition, they are also used to build up smaller, lighter and more efficient nano-sensors as scaffolds for tissues repair and cell growth [9] [10] [11]. To drive the drug delivery systems into the lymph-node cancer cells, synthesized MWCNTs with magnetite nano-particles were functionalized folic-acid and successfully loaded with Cisplatin (an anti-cancer drug) [12] [13]. Furthermore, CNTs have also been employed as drug delivery agents for treatment of hypertension (carvedilol) [14], asthma (theophylline) [15], human immunodeficiency virus (HIV) [16] and inflammation (dapsone, dexamethasone and ibuprofen) [17] [18] [19] [20] as well as some of brain diseases [21] [22], e.g., Alzheimer disease (AD) [23]. SWCNTs have recently been used as safe carriers to increase the effectiveness of AD treatment (against Mitchidoria in the brain) [23].

$\mathrm{AD}$ is the most common type of dementia, progressive and irreversible brain disorder that causes problems with behavior and slowly destroys thinking skills and memory. Its symptoms often develops gradually and gets worse overtime and classified into three stages; mild, moderate then becomes severe, which can be noticeably seen in the X-ray image shown in Figure 1, interfering with daily life tasks. $\mathrm{AD}$ has no current and effective cure so far to get rid of the progressive form of Alzheimer. Nowadays, treatment of AD symptoms are available and researchers still paying more attentions to find new techniques to slow worsening 


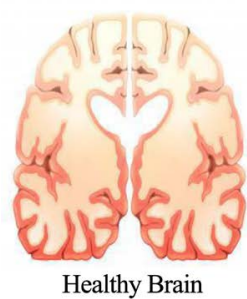

Healthy Brain
Alzheimer's Disease

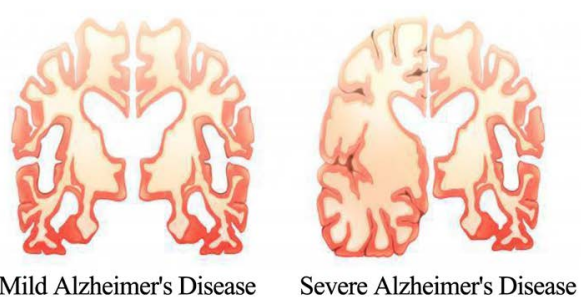

Figure 1. Schematic geametry (X-ray image) expose the difference between the health brain, and the mild and severe stages of Alzheimer disease.

of losing memory, improve quality of daily tasks and prevent Alzheimer's symptoms from developing. Yang et al. [23] who have shown that SWCNTs with diameter 0.8 to $1.6 \mathrm{~nm}$ and variant lengths $5-300 \mathrm{~nm}$ were successfully carried out with Acetylcholine ( $\mathrm{ACh}$ ) then directly delivered into brain, lysosomes are the targeted organelles of SWCNTs not mitochondria (MTCHD). This technique used the SWCNT as a catalyst due to its lack in the brain areas to increase the effectiveness of ACh drug. ACh can not be delivered directly into the brain with low doses because of its poor lipophilicity, this has recently been administrated that lack of ACh can be overcome by using SWCNT as carrier with high bio-safety [13]. Inside the brain, SWCNTs enter directly into the lysosomes, not to the MTCHD, which are the targeted organelles, but the high doses rise up the opportunity of SWCNTs to enter to the MTCHD. SWCNTs can be successfully exploited to deliver $\mathrm{ACh}$ into the targeted lysosomes to achieve therapeutic effects without undesired toxic effects [23].

The proposed model is designed to investigate the mechanism of encapsulation of two different antiviral compounds, Acetylcholine (used for the early stages) and Ravastigmine (RAV) (used for the moderate and severe stages) with chemical formulas $\mathrm{C}_{7} \mathrm{NH}_{16} \mathrm{O}_{2}^{+}$and $\mathrm{C}_{14} \mathrm{H}_{22} \mathrm{~N}_{2} \mathrm{O}_{2}$ as shown in Figure 2(a) and Figure 2(b), respectively, inside the SWCNTs of variant radii $r_{c}$. Firstly, we obtain the mathematical geometry for each antiviral compound and also evaluate the total energy arising form each antiviral compound encapsulated inside a SWCNT of radius $r_{c}$. ACh structure comprised as two-connected sphere with different radii $r_{s}$, while RAV modelled as two possible structres; an ellipsoid and cylinder, each interacting with the interior wall of SWCNTs with variant radii $r_{c}$. This paper is structured as, in the first section, we briefly outline the significance of usefulness of SWCNTs in many modern applications at nano-scales. Next, we apply the van der Waals force, Lennard-Jones potential and a discrete-continuum approximation to investigate the medical application which describes the possibility of conjugation between a SWCNT and antiviral compounds specialized to treat $\mathrm{AD}$ by inhibiting the growth of MTCDH (infected site in the brain). We also determine the magnitude of the potential energy arising from the interaction between antiviral compounds and nanotubes with various radii $r_{c}$. Followed by a discussion and analysis our results in section 3. Finally, conclusions and remarks are given in the last section. 
a) Acetylcholine Drug

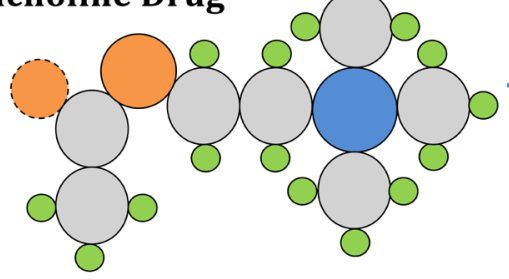

b) Ravastigmine Drug
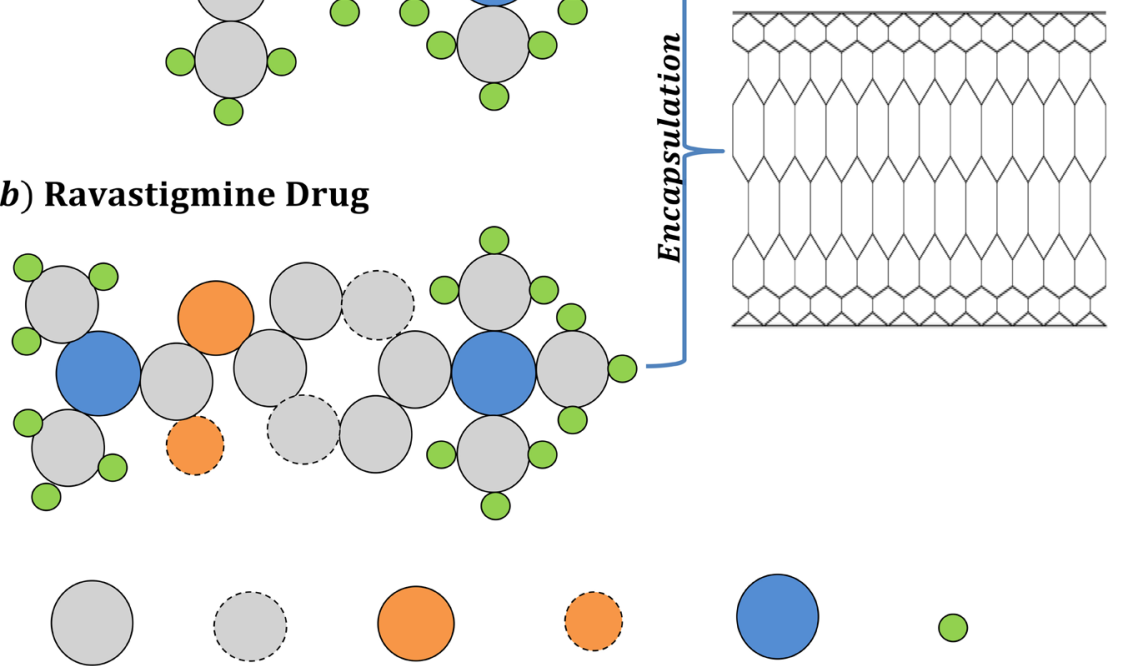

Od. $b .=0.70$

Ns. $b .=0.70$

Hs. b. $=0.37 \AA$

c) Schematic Geometry

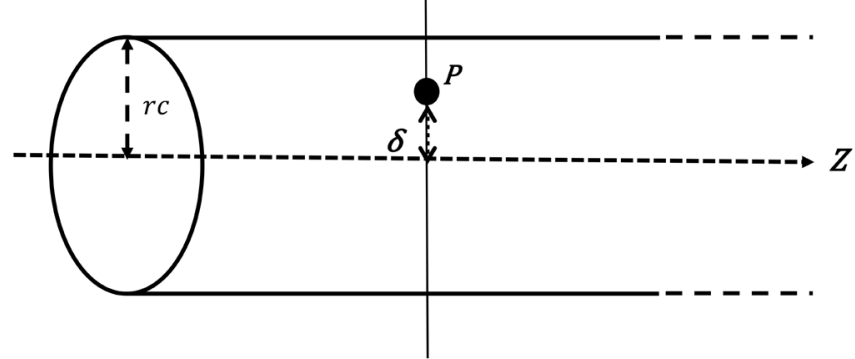

Figure 2. Schematic geometry for: (a) ACh antiviral compound as an interior atom interacting with a SWCNT of radius $r_{c}$ (sb:single bond and $d b$ :double bond); (b) RAV antiviral compound as an interior atom interacting with a SWCNT of radius $r_{c}$ (sb:single bond and $d b$ :double bond); (c) Antiviral compound as an interior atom at point $P$ off-setting from the central-axis by a distance $\delta$.

\section{Mathematical Model}

Here, we obtain the bio-physical model which describes the adsorption of two different drugs into SWCNTs with variant radii $r_{c}$ as a mathematical model by using van der Waals forces. We use the Lennard-Jones potential and the continuum approach together to model the encapsulation of these drugs inside a SWCNT. Next, we use the Cartesian coordinate $(x, y, z)$ as a reference system to model the two interacting molecules, the certain biomolecule and the cylindrical nanotube. We assume that the point at atom $P$ has coordinates $(\delta, 0,0)$ and the SWCNT defined as a cylindrical tube parameterized by $\left(r_{c} \cos \theta, r_{c} \sin \theta, z\right)$, where $0 \leq \delta \leq r_{c}, \quad 0 \leq r_{c} \leq 1,-\pi<\theta \leq \pi$ and $-L<z \leq L$ as shown in Figure 2(c). Therefore, the distance $\rho$ from point $P$ to typical 
surface element of the cylindrical tube given by

$$
\rho^{2}=\left(r_{c} \cos \theta-\delta\right)^{2}+r_{c}^{2} \sin ^{2} \theta+z^{2}=\left(r_{c}-\delta\right)^{2}-4 \delta r_{c} \sin ^{2}(\theta / 2)+z^{2} .
$$

The Lennard-Jones potential given as

$$
\beta(\rho)=\frac{-A}{\rho^{6}}+\frac{B}{\rho^{12}},
$$

where $\beta(\rho)$ is the potential function, $\rho$ denotes the distance between two molecular structures, and $A$ and $B$ are the attractive and repulsive constants. The physical parameters, $A=4 \varepsilon \sigma^{6}$ and $B=4 \varepsilon \sigma^{12}$, are calculated by using the empirical combining laws given by given by $\varepsilon_{i j}=\sqrt{\varepsilon_{i} \varepsilon_{j}}, \sigma_{i j}=\left(\sigma_{i}+\sigma_{j}\right) / 2$ and $\zeta_{i j}=\sqrt{\zeta_{i} \zeta_{j}}$, where $\varepsilon$ is the well depth, $\sigma$ is the van der Waals diameter and $\zeta$ is the non-bond energy [24] [25]. Here, we apply continuum approximation, atoms are assumed to be uniformly distributed over the surfaces of the two interacting molecules, to evaluate the interaction energy between two well-defined molecules by performing double integral over the surface of each molecule. From the work of Thamwattana et al. [26], the interaction energy is given by

$$
E_{a}=\eta_{c} \int_{V} \beta(\rho) \mathrm{d} V=\eta_{c} \int_{V}\left(-A I_{3}+B I_{6}\right) \mathrm{d} V
$$

where $\eta_{c}$ is the atomic surface densities of atoms on the nanotube and $\mathrm{d} V$ is a typical surface element located on the interacting molecule. The integral $I_{n}$ $(n=3,6)$ is defined by

$$
I_{n}=r_{c} \int_{-\infty}^{\infty} \int_{-\pi}^{\pi} \frac{1}{\left[\left(r_{c}-\delta\right)^{2}-4 \delta r \sin ^{2}(\theta / 2)+z^{2}\right]} \mathrm{d} \theta \mathrm{d} z,
$$

we may re-write the equation 4 by using the hypergeometric function as

$$
I_{n}=\frac{2 \pi}{r_{c}^{2 n-2}} B(n-1 / 2,1 / 2) \sum_{m=0}^{\infty}\left(\frac{(n-1 / 2)_{m} \delta^{m}}{m ! r_{c}^{m}}\right)^{2} .
$$

Next, we assume that the atom at point $P$ is within the volume element of each biomolecule. Thus, we can determine the molecular interaction arising from the certain drug by performing the volume integral of $E_{d}$ over the volume of the certain drug, namely

$$
\begin{aligned}
E_{d} & =\eta_{s} \int_{V} E_{a}(\delta) \mathrm{d} V \\
& =\eta_{s} \eta_{c} \int_{V}\left(-A I_{3}(\delta)+B I_{6}(\delta)\right) \mathrm{d} V \\
& =\eta_{s} \eta_{c}\left(-A K_{3}+B K_{6}\right),
\end{aligned}
$$

where $\delta$ is the distance from the nanotube axis to a typical point of the certain biomolecule and $\eta_{s}$ is the mean volume density of the biomolecule, which depends on the assumed configuration of the interacting biomolecule and $K_{n}$ can be given as

$$
K_{n}=\int_{V} I_{n}(\delta) \mathrm{d} V
$$




\subsection{Insertion of ACh as Two-Connected Spheres into SWCNT}

Here, we assume ACh structure modelled as two-connected spheres, the larger sphere centred at the origin point with radius $r_{s_{1}}$ and the samller sphere with radius $r_{s_{2}}$ located on the left side of the origin point. Each sphere assumed to be as a spherical shell parameterized $\left(r_{s} \cos \theta \sin \phi, r_{s} \sin \theta \sin \phi, r_{s} \cos \phi\right)$, where $-\pi<\theta \leq \pi, 0 \leq \phi \leq \pi, 0 \leq r_{s} \leq 1$ and $r_{s}$ is the radius of the spherical shell as shown in Figure 3(a). Further, the distance is given by $\rho^{2}=r_{s}^{2} r_{c}^{2} \sin ^{2} \phi$ and the spherical volume element is $\mathrm{d} V=r_{s} r_{c}^{2} \sin \phi \mathrm{d} r \mathrm{~d} \phi \mathrm{d} \theta$. From the work of Thamwattana et al. [26], the interaction energy between a spherical molecule and a cylindrical nanotube is given as

$$
E_{\text {Sphc-CNT }}=\eta_{c} \eta_{s}\left(-A D_{3}+B D_{6}\right)=\eta_{c} \eta_{s} \int_{V}\left(-A J_{3}+B J_{6}\right) \mathrm{d} V,
$$

where $\eta_{c}$ and $\eta_{s}$ are the atomic volume densities of the cylindrical nanotube and spheroidal molecule, respectively. So, the integral $J_{n} \quad(n=3,6)$ is given by

$$
J_{n}=\int_{-\pi}^{\pi} \int_{0}^{\pi} \int_{0}^{1} r_{s}^{2 n+2} r_{c}^{2 n+2} \sin ^{2 n+2} \phi \mathrm{d} r \mathrm{~d} \phi \mathrm{d} \theta,
$$

by using the relation between the beta and hypergeometric functions, $D_{n}$ can be expressed in terms of

$$
D_{n}=\frac{8 \pi^{2} r_{s}^{3}}{3 r_{c}^{2 n-2}} B(n-1 / 2,1 / 2) \sum_{m=0}^{\infty} \frac{(n-1 / 2)_{m}(n-1 / 2)_{m}}{(5 / 2)_{m} m !}\left(\frac{r_{s}^{2}}{r_{c}^{2}}\right)^{m} .
$$

\subsection{Insertion of RAV into SWCNT}

To evaluate the total energy arising from the RAV drug interaction with SWCNT of radius $r_{c}$, we consider two possible structures as models for RAV molecule which are an ellipsoid and cylinder as shown in Figure 3(b) and Figure 3(c), respectively.

\subsubsection{An Ellipsoid Model}

The RAV molecule assumed to be as a spheroidal structure, parameterized by $(\operatorname{ar} \sin \phi \cos \theta, a r \sin \phi \sin \theta, b r \cos \phi)$, where $0 \leq r \leq 1,-\pi<\theta \leq \pi, 0 \leq \phi \leq \pi$, and $a$ and $b$ are the equatorial semi-axis length and polar semi-axis length (along the $z$-axis) of spheroidal structure, respectively, as shown in Figure 3(b). Further, the distance is given by $\rho^{2}=a^{2} r^{2} \sin ^{2} \phi$ and the spheroidal volume element is $\mathrm{d} V=a^{2} b r^{2} \sin \phi \mathrm{d} r \mathrm{~d} \phi \mathrm{d} \theta$. From the work of Thamwattana et al. [26], the interaction energy between a spheroidal molecule and a cylindrical nanotube is given as

$$
E_{\text {Sphd-CNT }}=\eta_{c} \eta_{l}\left(-A T_{3}+B T_{6}\right)=\eta_{c} \eta_{l} \int_{V}\left(-A W_{3}+B W_{6}\right) \mathrm{d} V,
$$

where $\eta_{l}$ is the mean volume density of the spheroidal molecule, respectively. So, the integral $W_{n} \quad(n=3,6)$ is given by

$$
W_{n}=\int_{-\pi}^{\pi} \int_{0}^{\pi} \int_{0}^{1} a^{2 n+2} b r^{2 n+2} \sin ^{2 n+2} \phi \mathrm{d} r \mathrm{~d} \phi \mathrm{d} \theta .
$$

The Integral $T_{n}$ can be expressed in terms of 

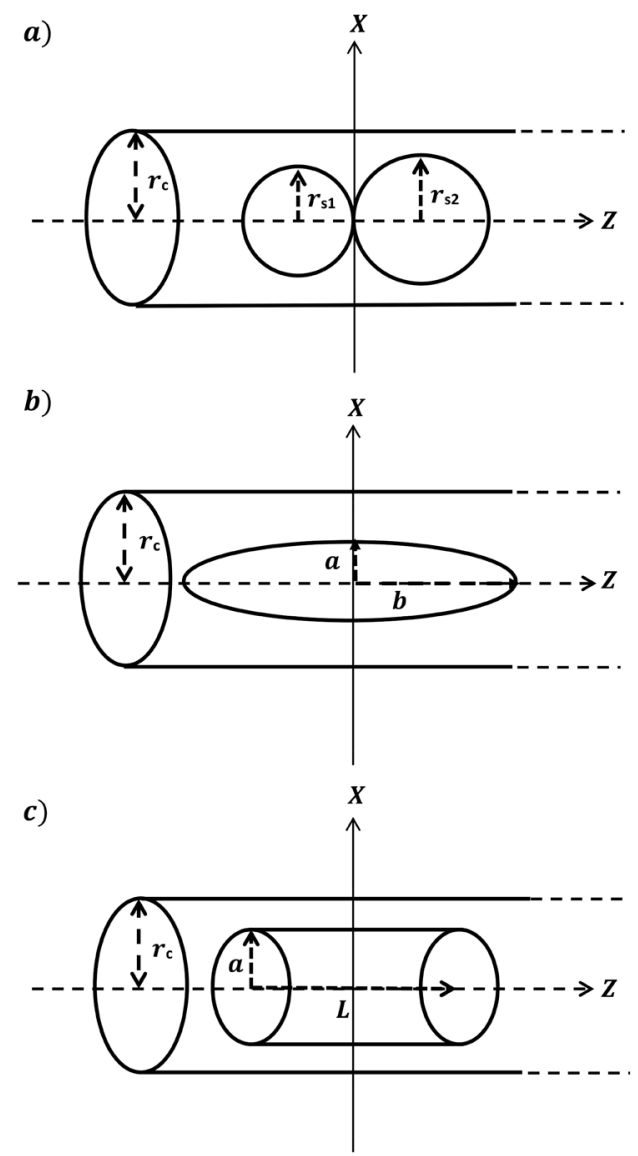

Figure 3. The possible configuration for each antiviral compound: (a) ACh molecule splitted as two-connected sphere located on the left and right sides of the origin point; (b) RAV molecule as an ellipsoid structure; (c) RAV molecule as a perfect cylinder, each configuration interacting with a SWCNT of radius $r_{c}$.

$$
T_{n}=\frac{8 \pi^{2} a^{2} b}{3 r_{c}^{2 n-2}} B(n-1 / 2,1 / 2) \sum_{m=0}^{\infty} \frac{(n-1 / 2)_{m}(n-1 / 2)_{m}}{(5 / 2)_{m} m !}\left(\frac{a^{2}}{r_{c}^{2}}\right)^{m} .
$$

To obtain and evaluate the interaction energy for each configuration as shown in Figure 3, we need to determine the potential energy arising from the specific atom at point $P$ inside the cylindrical nanotube as shown in Figure 2(c) (this atom is withing the volume of RAV).

\subsubsection{Cylindrical Model}

Here, we model the RAV molecule modelled as a perfect cylinder located at the origin (centered) with radius $a$ and length $L=2 b$ as shown in Figure 3(c). A typical point in the cylinder can be parameterized by $(\operatorname{arcos} \theta, \operatorname{arsin} \theta, z)$, where $0 \leq r \leq 1,-\pi \leq \theta \leq \pi$ and $-L \leq z \leq L$. Therefore, the distance $\delta$ is given by $\delta^{2}=a^{2} r^{2}$ and the volume element of cylinder is $\mathrm{d} V=a^{2} r \mathrm{~d} r \mathrm{~d} \theta \mathrm{d} z$. From Thamwattana's work et al. [26], the interaction energy between a cylindrical molecule and a cylindrical nanotube given as

$$
E_{\text {Cyld-CNT }}=\eta_{c} \eta_{d}\left(-A Y_{3}+B Y_{6}\right)=\eta_{c} \eta_{d} \int_{V}\left(-A G_{3}+B G_{6}\right) \mathrm{d} V,
$$


where $\eta_{d}$ is the mean volume density of the cylindrical molecule. So, the integral $G_{n}(n=3,6)$ is given by

$$
G_{n}=\int_{-L}^{L} \int_{-\pi}^{\pi} \int_{0}^{1} a^{2 n+2} r^{2 n+2} \mathrm{~d} r \mathrm{~d} \theta \mathrm{d} z
$$

So, $Y_{n}$ is given as

$$
Y_{n}=\frac{4 \pi^{2} a^{2} L}{r_{c}^{2 n-2}} B(n-1 / 2,1 / 2) \sum_{m=0}^{\infty} \frac{(n-1 / 2)_{m}(n-1 / 2)_{m}}{(2)_{m} m !}\left(\frac{a^{2}}{r_{c}^{2}}\right)^{m} .
$$

\section{Results and Discussion}

In this section, we apply Lennard-Jones potential and the discrete-continuum approach to evaluate the interaction energy of each drug interacting inside SWCNTs with variant radii $r_{c}$. The non-bond energy, well-depth $\varepsilon$ and van der Waals diameter $\sigma$ are shown in Table 1 . The physical parameters and illustrated radii $r_{c}$ of CNTs are given in Table 2. The attractive and repulsive constants are calculated by using the combining laws ( $A=4 \varepsilon \sigma^{6}$ and $B=4 \varepsilon \sigma^{12}$ ) and are given in Table 3 . The volume density for each configuration calculated as the total number of atoms that are containing the specific molecule are divided by the volume of the molecule structure, spherical shape $\left(\eta_{s}\right)$, spheroidal structure $\left(\eta_{l}\right)$ and cylindrical shell $\left(\eta_{d}\right)$, which are $\eta_{s}=26 /\left(4 \pi r_{s}^{3} / 3\right), \quad \eta_{l}=31 /\left(4 \pi a^{2} b / 3\right)$ and $\eta_{d}=31 /\left(2 \pi a^{2} L\right)$, respectively. Next, we evaluate and plot the minimum energies (for all configurations) which are arising from the ACh-SWCNT and RAV-SWCNT interactions. We also deduce the critical radius of SWCNT that will accept both antiviral compounds (ACh-SWCNT and RAV-SWCNT). The minimum energies for all configurations are obtained based on the equilibrium position of each molecule being away from the interior wall of CNT and its radius $r_{c}$ along the range of $\mathrm{z}$-axis. In this model, we observe the encapsulation of ACh and RAV inside the nanotubes with radius in the range $3.204 \AA<r_{c}<7.551 \AA$ and the minimum energies obtained for both configurations when $r_{c}$ greater than $3.391 \AA$. The lowest interaction energy for ACh-SWCNT and RAV-SWCNT interactions is obtained when the radius of nanotube in the range $3.86 \AA<r_{c}<4.07 \AA$ as shown in Figures 4-6.

For the three proposed configurations, we note that the both antiviral compounds, ACh-SWCNT and RAV-SWCNT, are repulsive and unstable when $r_{c}<3.325$ and $r_{c}<3.391 \AA$, respectively, and the $(9,2)$ SWCNT of radius $r_{c}=3.973 \AA$ is the most favorable nanotube followed by the condition where $r_{c}=3.861,3.775,4.615,3.590,3.523,5.523,6.102,7.551$ and $3.391 \AA$, respectively. For all interactions, ACh-SWCNT (connected-spheres), RAV-SWCNT (spheroidal) and RAV-SWCNT (cylindrical), are with minimum energies of approximately $-0.664,-1.059$ and $1.204 \mathrm{kcal} / \mathrm{mol}$, respectively. Furthermore, we can noticeably see that the magnitude of the minimum energy for RAV-SWCNT (an ellipsoid structure) interaction is slightly smaller than that of RAV-SWCNT (perfect cylinder). We also note that the perfect cylinder (RAV) has the 
Table 1. The Lennard-Jones constants ( $\varepsilon$. Bond length, $\sigma$. Non-bond distance and $\zeta$ Non-bond energy) (single bond: sb, double bond: $\mathrm{db}$ ) [24] [27] [28].

\begin{tabular}{cccccccc}
\hline Interaction & $\mathcal{\varepsilon}(\AA)$ & $\sigma(\AA)$ & $\zeta(\mathrm{Kcal} / \mathrm{mol})$ & Interaction & $\mathcal{\varepsilon}(\AA)$ & $\sigma(\AA)$ & $\zeta(\mathrm{Kcal} / \mathrm{mol})$ \\
\hline $\mathrm{H}-\mathrm{H}$ & 0.74 & 2.886 & 0.044 & $\mathrm{O}-\mathrm{H}$ & 0.96 & 3.193 & 0.051 \\
$\mathrm{O}-\mathrm{O}(\mathrm{sb})$ & 1.48 & 3.500 & 0.060 & $\mathrm{O}-\mathrm{O}(\mathrm{db})$ & 1.21 & 3.500 & 0.060 \\
$\mathrm{~N}-\mathrm{N}$ & 1.45 & 3.660 & 0.069 & $\mathrm{~N}-\mathrm{H}$ & 1.00 & 3.273 & 0.055 \\
$\mathrm{C}-\mathrm{C}(\mathrm{sb})$ & 1.54 & 3.851 & 0.105 & $\mathrm{C}-\mathrm{H}$ & 1.09 & 3.368 & 0.068 \\
$\mathrm{C}-\mathrm{C}(\mathrm{db})$ & 1.34 & 3.851 & 0.105 & $\mathrm{C}-\mathrm{O}(\mathrm{sb})$ & 1.43 & 3.675 & 0.079 \\
$\mathrm{C}-\mathrm{O}(\mathrm{db})$ & 1.20 & 3.675 & 0.079 & $\mathrm{C}-\mathrm{N}$ & 1.47 & 3.755 & 0.085 \\
\hline
\end{tabular}

Table 2. Parameters for carbon nanotubes, ACh and RAV molecules.

\begin{tabular}{|c|c|c|c|}
\hline Radius of CNT $(7,2)$ & $3.204 \AA$ [29] & Radius of CNT $(8,1)$ & $3.325 \AA[29]$ \\
\hline Radius of CNT $(5,5)$ & $3.390 \AA$ [29] & Radius of CNT $(9,0)$ & $3.523 \AA$ [29] \\
\hline Radius of CNT $(8,2)$ & $3.591 \AA[29]$ & Radius of CNT $(7,4)$ & $3.775 \AA$ [29] \\
\hline Radius of CNT $(8,3)$ & $3.861 \AA[29]$ & Radius of CNT $(9,2)$ & $3.973 \AA$ [29] \\
\hline Radius of CNT $(10,3)$ & $4.615 \AA[29]$ & Radius of CNT $(13,2)$ & $5.523 \AA[29]$ \\
\hline Radius of CNT $(9,9)$ & $6.102 \AA[29]$ & Radius of CNT $(14,8)$ & $7.551 \AA[29]$ \\
\hline $\begin{array}{l}\text { Radius of the smaller } \\
\text { sphere }\end{array}$ & $r_{s_{1}}=2.03 \AA$ & $\begin{array}{c}\text { Radius of the larger } \\
\text { sphere }\end{array}$ & $r_{s_{2}}=2.52 \AA$ \\
\hline $\begin{array}{l}\text { Equatorial-axes of the } \\
\text { spheroidal molecule }\end{array}$ & $a=2.54 \AA$ & $\begin{array}{l}\text { Polar semi-axes of the } \\
\text { spheroidal molecule }\end{array}$ & $b=6.205 \AA$ \\
\hline $\begin{array}{l}\text { Length of cylinder } \\
\text { molecule }\end{array}$ & $L=2 b=12.41 \AA$ & $\begin{array}{c}\text { Surface density for the } \\
\text { SWCNT }\end{array}$ & $\eta_{c}=0.381 \AA^{-2}$ \\
\hline $\begin{array}{l}\text { Volume density for the } \\
\text { larger sphere }\end{array}$ & $\eta_{s_{1}}=0.2388 \AA^{-3}$ & $\begin{array}{l}\text { Volume density for the } \\
\text { smaller sphere }\end{array}$ & $\eta_{s_{2}}=0.2856 \AA^{-3}$ \\
\hline $\begin{array}{l}\text { Volume density for the } \\
\text { spheroidal molecule }\end{array}$ & $\eta_{l}=0.1849 \AA^{-3}$ & $\begin{array}{l}\text { Volume density for the } \\
\text { cylindrical molecule }\end{array}$ & $\eta_{d}=0.0617 \AA^{-3}$ \\
\hline
\end{tabular}

Table 3. Numerical values of the significant constants $(A$ and $B)$ involved in this model.

\begin{tabular}{ccccc}
\hline Interaction & Attractive & Value $\left(\AA^{6} \mathrm{kcal} / \mathrm{mol}\right)$ & Repulsive & $\begin{array}{c}\text { Value }\left(\AA^{12} \times 10^{3}\right. \\
\mathrm{kcal} / \mathrm{mol})\end{array}$ \\
\hline ACh & $A_{\mathrm{ACh}}$ & 23.38 & $B_{\mathrm{ACh}}$ & 54.386 \\
$\mathrm{RAV}$ & $A_{\mathrm{RAV}}$ & 23.43 & $B_{\mathrm{RAV}}$ & 56.598 \\
$\mathrm{CNT}$ & $A_{\mathrm{CNT}}$ & 17.40 & $B_{\mathrm{CNT}}$ & 29.000 \\
$\begin{array}{c}\text { Spherical shell } \\
\left(r_{s_{1}}=2.52\right)\end{array}$ & $A_{s_{1}}$ & 23.52 & $B_{s_{1}}$ & 54.825 \\
$\begin{array}{c}\text { Spherical shell } \\
\left(r_{s_{2}}=2.03\right)\end{array}$ & $A_{s_{2}}$ & 23.24 & $B_{s_{2}}$ & 53.946 \\
$\begin{array}{c}\text { An ellipsoid } \\
\text { configuration (RAV) }\end{array}$ & $A_{l}$ & 23.43 & & 56.598 \\
$\begin{array}{c}\text { Cylindrical } \\
\text { configuration (RAV) }\end{array}$ & $A_{c}$ & 23.43 & $B_{l}$ & \\
\hline
\end{tabular}



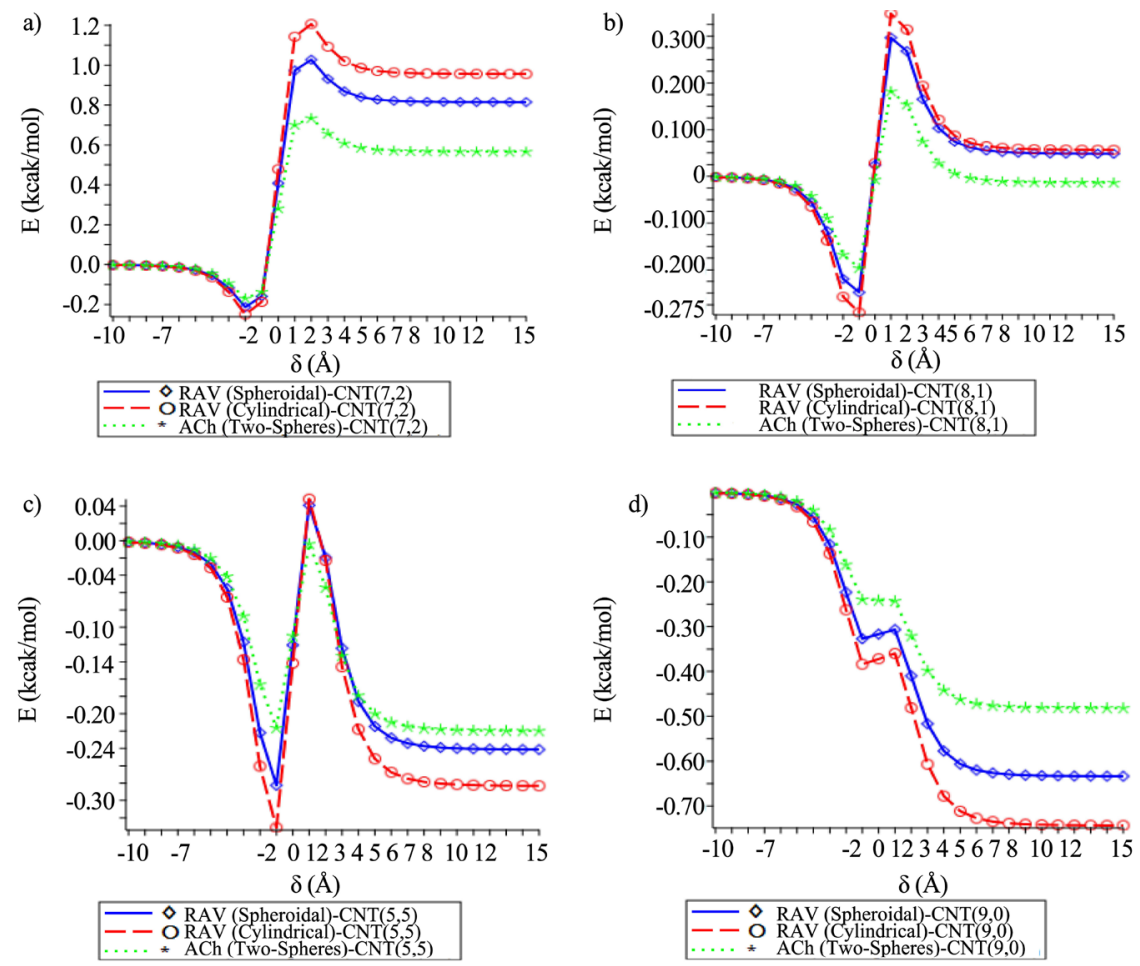

Figure 4. Interaction energy $(E)$ arising from the encapsulation of two antiviral compounds (ACh and RAV) inside SWCNTs with varian radii $r_{c}$ a) $r_{c}=3.204 \mathrm{~b}$ ) $r_{c}=3.325$ c) $r_{c}=3.391$ d) $r_{c}=3.523 \AA$.
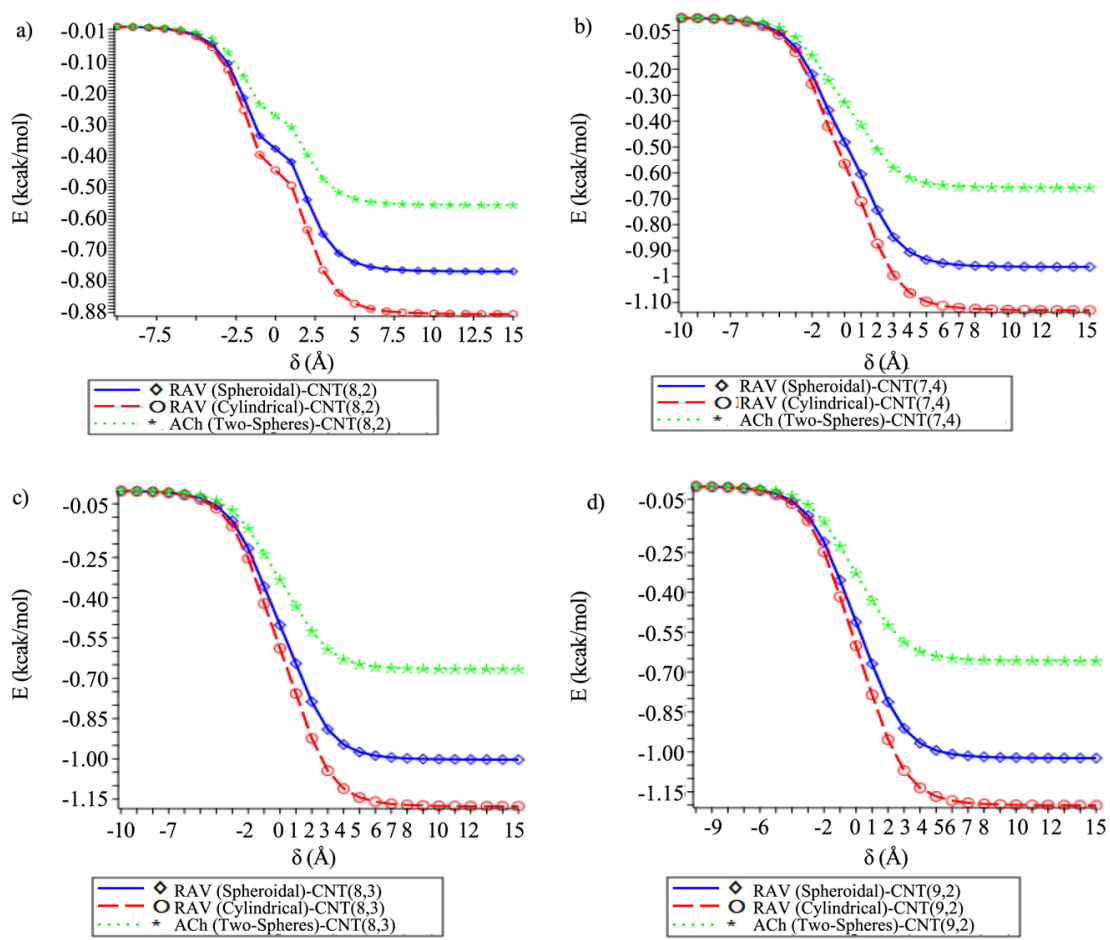

Figure 5. Interaction energy $(E)$ arising from the encapsulation of two antiviral compounds (ACh and RAV) inside SWCNTs with varian radii $r_{c}$ a) $r_{c}=3.590 \mathrm{~b}$ ) $r_{c}=3.775$ c) $r_{c}=3.861$ d) $r_{c}=3.973 \AA$. 

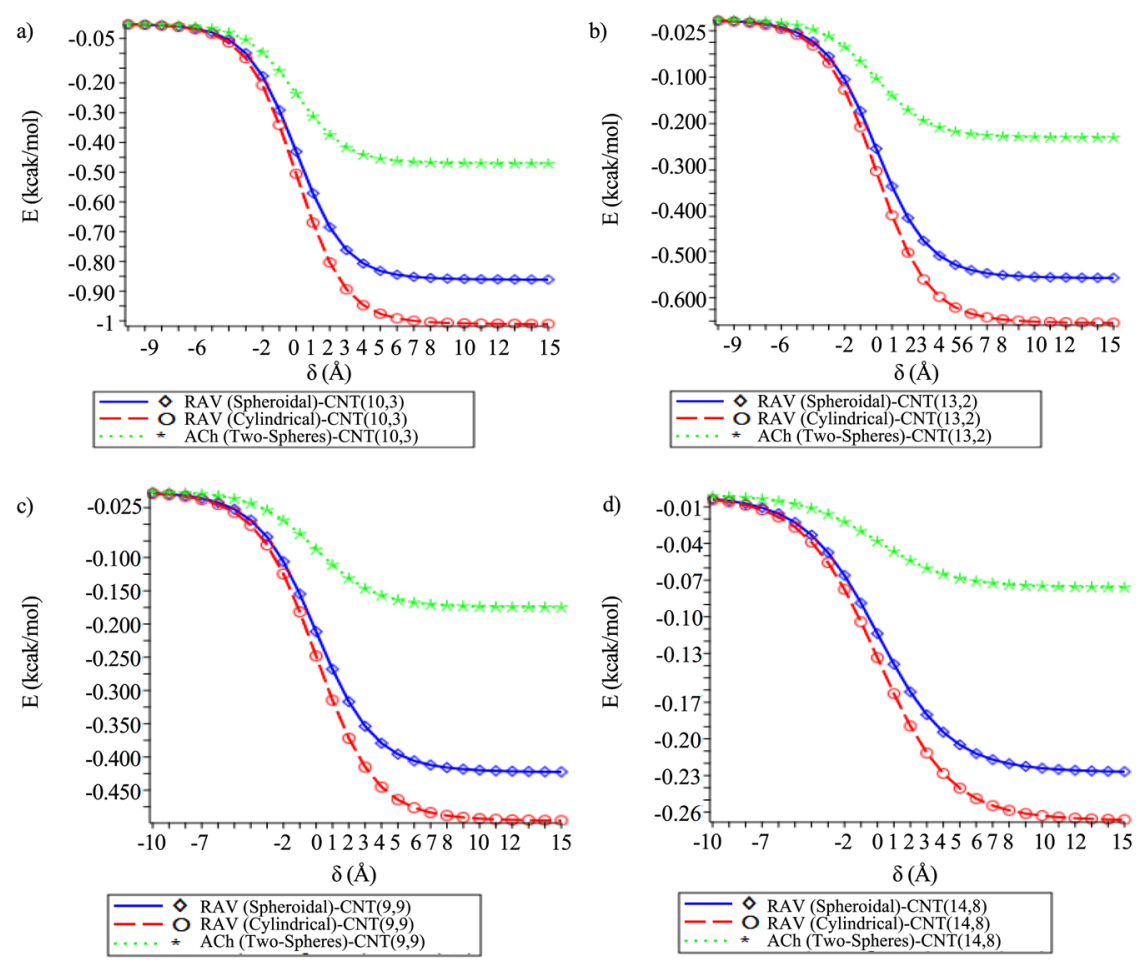

Figure 6. Interaction energy $(E)$ arising from the encapsulation of two antiviral compounds (ACh and RAV) inside SWCNTs with varian radii $r_{c}$ a) $r_{c}=4.615$ b) $r_{c}=5.523$ c) $r_{c}=6.102$ d) $r_{c}=7.551 \AA$.

maximum binding energy because of its volume $502.804 \AA^{3}$ being larger than that of an ellipsoid structure (RAV) which is $167.601 \AA^{3}$. This means that smaller size of an ellipsoid ends requires smaller size of nanotube to accommodate the spheroidal shell (RAV) compared with that of cylindrical structure (RAV) despite having similar dimensions. Moreover, we observe that our results consistently agree with the most recent research findings, for example, Dresselhaus et al. [1] predict that the $(5,5) \mathrm{CNT}$ of $r_{c}=3.391$ could be the most significant and smallest effective physical nanotube, and ACh drug can be carried with SWCNT of radius in the range of $4 \AA \leq r_{c} \leq 8 \AA$ ( $8 \AA<$ diameter = $2 r_{c}<16 \AA$ ) delivered to the target and infected cells [23].

\section{Conclusion}

In this study, the Lennard-Jones potential and continuum approach are adopted to evaluate the minimum energy for each configuration. The proposed model obtained mathematically by representing each molecule using the rectangular coordinate $(x, y, z)$ as a reference system. Through investigation, we find that the SWCNT plays a significant role by increasing the effectiveness of the antiviral compounds against the growth and symptoms of the AD. The SWCNT is a selective tool because of its distinct properties, such as high conductivity and low solubility in aqueous media. It can be concluded that the RAV antiviral compound is more effective against the $\mathrm{AD}$ growth, and both antiviral 
compounds, ACh and RAV, would not be accepted when $r_{c}<3.391 \AA$. For all possible configurations, we note that the lowest minimum energy obtained when $r_{c}=3.973 \AA$ A. Our results are in very good agreement with Yang's work who has shown that the ACh antiviral compound is successfully carried out and conjugated with SWCNTs with variant radii $r_{c}$ [23].

\section{Acknowledgements}

The author acknowledges financial support from the Research and Consultation Centre (RCC) at the University of Business and Technology.

\section{Funding}

This project funded by the Research and Consultation Centre (RCC) at the University of Business and Technology.

\section{Conflicts of Interest}

Has no conflict of Interest.

\section{Ethical Approval}

This article does not contain any studies with animals performed by any of authors.

\section{References}

[1] Dresselhaus, M.S., Dresselhaus, G. and Eklund, P.C. (1996) Science of Fullerenes and Carbon Nanotubes. Academic Press, San Diego, CA.

[2] Pantarotto, D., Partidos, C.D., Graff, R., Hoebeke, J., Briand, J.P., Prato, M. and Bianco, A. (2003) Synthesis, Structural Characterization, and Immunological Properties of Carbon Nanotubes Functionalized with Peptides. Journal of the American Chemical Society, 125, 6160-6164. https://doi.org/10.1021/ja034342r

[3] De Jong, W.H. and Borm, P.J.A. (2008) Drug Delivery and Nanoparticles: Applications and Hazards. International Journal of Nanomedicine, 3, 133-149. https://doi.org/10.2147/IJN.S596

[4] Vardharajula, S., Ali, S.Z., Tiwari, P.M., Eroglu, E., Vig, K., Dennis, V.A. and Singh, S.R. (2012) Functionalized Carbon Nanotubes: Biomedical Applications. International Journal of Nanomedicine, 7, 5361-5374.

[5] Monthioux, M. and Kuznetsov, V.L. (2006) Who Should Be Given the Credit for the Discovery of Carbon Nanotubes? Carbon, 44, 1612-1623. https://doi.org/10.1016/j.carbon.2006.03.019

[6] Iijima, S. (1991) Helical Microtubules of Graphitic Carbon. Nature, 354, 56-58. https://doi.org/10.1038/354056a0

[7] Donaldson, K., Poland, C.A., Murphy, F.A., MacFarlane, M. and Chernova, T. (2013) Pulmonary Toxicity of Carbon Nanotubes and Asbestos-Similarities and Differences. Advanced Drug Delivery Reviews, 65, 2078-2086. https://doi.org/10.1016/j.addr.2013.07.014

[8] Li, J., Pant, A., Chin, C.F., Ang, W.H., Menard-Moyon, C., Nayak, T.R., Gibson, D., Ramaprabhu, S., Panczyk, T. and Bianco, A. (2014) In Vivo Biodistribution of Pla- 
tinum-Based Drugs Encapsulated into Multi-Walled Carbon Nanotubes. Nanomedicine: Nanotechnology, Biology and Medicine, 10, 1465-1475.

https://doi.org/10.1016/j.nano.2014.01.004

[9] Heister, E., Brunner, E.W., Dieckmann, G.R., Jurewicz, I. and Dalton, A.B. (2013) Are Carbon Nanotubes a Natural Solution? Applications in Biology and Medicine. ACS Applied Materials \& Interfaces, 5, 1870-1891. https://doi.org/10.1021/am302902d

[10] Shin, S.R., Bae, H., Cha, J.M., Mun, J.Y., Chen, Y.C., Tekin, H., Shin, H., Farshchi, S., Dokmeci, M.R. and Tang, S. (2012) Carbon Nanotube Reinforced Hybrid Microgels as Scaffold Materials for Cell Encapsulation. ACS Nano, 6, 362-372. https://doi.org/10.1021/nn203711s

[11] De la Zerda, A., Zavaleta, C., Keren, S., Vaithilingam, S., Bodapati, S., Liu, Z., Levi, J., Smith, B.R., Ma, T.J., Oralkan, O., Cheng, Z., Chen, X., Dai, H., Khuri-Yakub, B.T. and Gambhir, S.S. (2008) Carbon Nanotubes as Photoacoustic Molecular Imaging Agents in Living Mice. Nature Nanotechnology, 3, 557-562. https://doi.org/10.1038/nnano.2008.231

[12] Yang, F., Fu, D.L., Long, J. and Ni, Q.X. (2008) Magnetic Lymphatic Targeting Drug Delivery system Using Carbon Nanotubes. Medical Hypotheses, 70, 765-767. https://doi.org/10.1016/j.mehy.2007.07.045

[13] Yang, Y.J., Tao, X., Hou, Q. and Chen, J.F. (2009) Fluorescent Mesoporous Silica Nanotubes Incorporating CdS Quantum Dots for Controlled Release of Ibuprofen. Acta Biomaterialia, 5, 3488-3496. https://doi.org/10.1016/j.actbio.2009.05.002

[14] Li, Y., Wang, T., Wang, J., Jiang, T., Cheng, G. and Wang, S. (2011) Functional and Unmodified MWNTs for Delivery of the Water-Insoluble Drug Carvedilol-A Drug-Loading Mechanism. Applied Surface Science, 257, 5663-5670. https://doi.org/10.1016/j.apsusc.2011.01.071

[15] Zhang, C.H., Luo, Y.L., Chen, Y.S., Wei, Q.B. and Fan, L.H. (2009) Preparation and Theophylline Delivery Applications of Novel PMAA/MWCNT-COOH Nanohybrid Hydrogels. Journal of Biomaterials Science, Polymer Edition, 20, 1119-1135. https://doi.org/10.1163/156856209X444466

[16] Al Garalleh, H., Thamwattana, N., Cox, B.J. and Hill, J.M. (2015) Modelling Interaction between $\mathrm{C}_{60}$ Antiviral Compounds and HIV Protease. Bulletin of Mathematical Biology, 77, 184-201. https://doi.org/10.1007/s11538-014-0056-2

[17] Vukovic, G.D., Tomic, S.Z., Marinkovic, A.D., Radmilovic, V., Uskokovic, P.S. and Colic, M. (2010) The Response of Peritoneal Macrophages to Dapsone Covalently Attached on the Surface of Carbon Nanotubes. Carbon, 48, 3066-3078. https://doi.org/10.1016/j.carbon.2010.04.043

[18] Naficy, S., Razal, J.M., Spinks, G.M. and Wallace, G.G. (2009) Modulated Release of Dexamethasone from Chitosan-Carbon Nanotube Films. Sensors and Actuators A: Physical, 155, 120-124. https://doi.org/10.1016/j.sna.2009.07.021

[19] Murakami, T., Ajima, K., Miyawaki, J., Yudasaka, M., Iijima, S. and Shiba, K. (2004) Drug-Loaded Carbon Nanohorns: Adsorption and Release of Dexamethasone in Vitro. Molecular Pharmaceutics, 1, 399-405. https://doi.org/10.1021/mp049928e

[20] Yang, F., Hu, J., Yang, D., Long, J., Luo, G., Jin, C., Yu, X., Xu, J., Wang, C. and Ni, Q.X. (2009) Pilot Study of Targeting Magnetic Carbon Nanotubes to Lymph Nodes. Nanomedicine, 4, 317-330. https://doi.org/10.2217/nnm.09.5

[21] Li, S., Lin, C., Wei, K., Huang, C., Hsu, P., Liu, H., Lu, Y., Lin, S., Yang, H. and Ma, C. (2016) Non-Invasie Screening for Early Alzheimer's Disease Diagnosis by Sensitively Immunomagnetic Biosensor. Scientific Reports, 6, Article No. 25155. 
https://doi.org/10.1038/srep25155

[22] Wang, J.T.W. and Al-Jamal, K.T. (2015) Functionalized Carbon Nanotubes: Revolution in Brain Delivery. Nanomedicine (London), 10, 2639-2642.

https://doi.org/10.2217/nnm.15.114

[23] Yang, Z., Zhang, Y., Yang, Y., Sun, L., Han, D., Li, H. and Wang, C. (2010) Pharmacological and Toxicological Target Organelles and Safe Use of Single-Walled Carbon Nanotubes as Drug Carriers in Treating Alzheimer Disease. Nanomedicine: Nanotechnology, Biology and Medicine, 6, 427-441. https://doi.org/10.1016/j.nano.2009.11.007

[24] Cottrell, L.T. (1958) The Strengths of Chemical Bonds. Butterworths Scientific Publications, London.

[25] Hirschfelder, J.O., Curtiss, C.F. and Byron, R.B. (1964) Molecular Theory of Gases and Liquids. Society for Industrial and Applied Mathematics, University of Winsconsin, Madison.

[26] Thamwattana, N., Baowan, D. and Cox, B.J. (2013) Modelling Bovine Serum Albumin inside Carbon Nanotubes. RSC Advances, 3, 23482-23488. https://doi.org/10.1039/c3ra43991g

[27] Rappi, A.K., Casewit, C.J., Colwell, K.S., Goddard III, W.A. and Skid, W.M. (1992) UFF, a Full Periodic Table Force Field for Molecular Mechanics and Molecular Dynamics Simulations. Journal of the American Chemical Society, 114, 10024-10035. https://doi.org/10.1021/ja00051a040

[28] Benson, S.W. (1965) III-Bond Energies. Journal of Chemical Education, 42, 502. https://doi.org/10.1021/ed042p502

[29] Dresselhaus, M.S., Dresselhaus, G. and Satio, R. (1995) Physics of Carbon Nanotubes. Carbon, 33, 883-891. https://doi.org/10.1016/0008-6223(95)00017-8 